\title{
Soft QCD Measurements at LHC - Status and Outlook
}

\author{
Jan Fiete Grosse-Oetringhaus* \\ CERN \\ E-mail: jgrosseodcern.ch
}

The first run of the LHC (2009-2012) has resulted in a tremendous legacy of soft physics results measured by ALICE, ATLAS, CMS, LHCb, LHCf and TOTEM. These measurements are crucial for successful phenomenological understanding and modelling of the underlying processes with the ultimate goal to learn about soft QCD. A selection of important topics is reviewed including particle multiplicities, underlying-event distributions, multiple-parton interactions, identifiedparticle abundances and signatures of collectivity in small systems. Where available, first results from the second run of LHC at a center-of-mass energy of $13 \mathrm{TeV}$ are included.

XXVII International Symposium on Lepton Photon Interactions at High Energies 17-22 August 2015

Ljubljana, Slovenia

${ }^{*}$ Speaker. 


\section{Introduction}

The strong interaction is one of the four fundamental forces observed in nature. The underlying theory, Quantum Chromodynamics (QCD), is one of the building blocks for the Standard Model which is almost completely describing particles and their interactions. QCD is a very successful theory and calculates a large number of observables to significant precision, e.g. jet production at high $p_{\mathrm{T}}$. In the energy regime where the strong coupling $\alpha_{\mathrm{S}}$ is small, perturbative methods can be applied (pQCD) and thus calculations are precise. In the soft regime, however, perturbative methods are not applicable and the understanding of soft QCD builds on phenomenology and modelling. Although cross-sections for hard processes at LHC are larger than at any other collider, most of the processes occurring at LHC are still in the soft regime: parton-parton scatterings with a momentum transfer of a few $\mathrm{GeV} / c$. In consequence, measurements of soft processes at LHC are crucial for successful phenomenological understanding and modelling with the ultimate goal to learn about soft QCD. In addition, the particle produced in soft processes represent a significant background for measurements of rare probes and their understanding is a precondition for trigger strategies, analysis methods and precision measurements for rare probes.

The first run of the LHC (2009-2012) has resulted in a tremendous legacy of soft physics results measured by ALICE, ATLAS, CMS, LHCb, LHCf and TOTEM. Details can be found on the public result pages of the collaborations: [1-6]. In the following, a selection of important topics is reviewed.

\section{Particle Multiplicities}

The measurement of particle abundances is an elementary characterization of particle collisions [7]. Typical observables are the pseudorapidity density $d N_{\mathrm{ch}} / d \eta$ (the average number of particles as a function of pseudorapidity $\eta$ ), the transverse momentum distribution $d N_{\mathrm{ch}} / d p_{\mathrm{T}}$ (the average number of particles as a function of $p_{\mathrm{T}}$ ) and the multiplicity distribution $P\left(N_{\mathrm{ch}}\right)$ (the probability distribution for the occurrence of a collisions with $N_{\mathrm{ch}}$ particles). In addition, the correlation of the average transverse momentum $\left\langle p_{\mathrm{T}}\right\rangle$ with the event multiplicity allows to probe the dynamics of the collisions. While these definitions are simple, it is important to consider that these observables can be measured in different event classes and in different phase-space regions. The experiments at LHC provide results for inelastic and non-single diffractive collisions as well as in event classes with a particle-level definition such as events with at least one charged particle in a certain $\eta$ - and $p_{\mathrm{T}}$-window. The former involves usually larger model-dependent extrapolations but allows easier model comparisons and comparisons to other center-of-mass energies, while the latter has smaller overall uncertainties.

Figure 1 shows examples of these measurements. Further measurements from LHC can be found in [8-24] which span a significant fraction of the phase space of particles produced in pp collisions at the LHC as also LHCb and TOTEM contribute with their forward capabilities. Comparisons of the results between the experiments show good agreement. At the time of the first measurements at LHC, the results differed significantly from model predictions, in particular the overall multiplicity was underestimated substantially as well as events with large multiplicities were found more often in data than in model predictions (see e.g. [12, 18]). Model development 

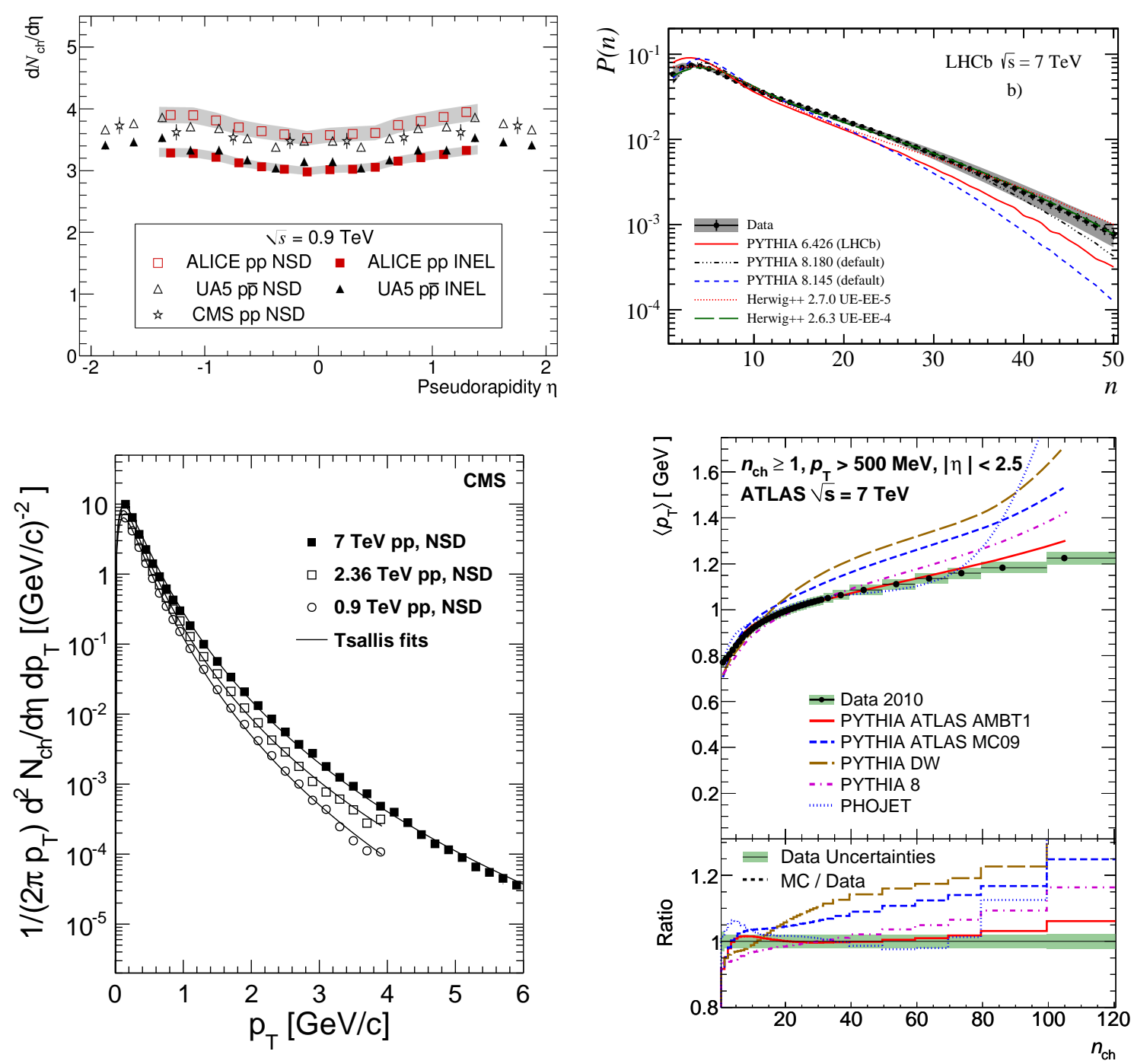

Figure 1: Examples of pseudorapidity density $d N_{\mathrm{ch}} / d \eta$ [17] (top left), transverse momentum distribution $d N_{\mathrm{ch}} / d p_{\mathrm{T}}$ [11] (bottom left), multiplicity distribution $P\left(N_{\mathrm{ch}}\right)$ [15] (top right) and $\left\langle p_{\mathrm{T}}\right\rangle$ as a function of event multiplicity [8] (bottom right) measurements.

and tuning effort has led in the meanwhile to a good descriptions of these observables; see [25] for a recent review.

First measurements are also available at $\sqrt{s}=13 \mathrm{TeV}$ [26-28] and examples are shown in Fig. 2. It is noteworthy that EPOS LHC [29] as well as PYTHIA 8 with the Monash tune [30] reproduce the measurements within about $10 \%$.

\section{Underlying-Event Distributions}

Further quantification of the dynamics of particle collisions is obtained by studying the underlying event referring to the activity below the hardest scattering in the event. In practice the event is split into three regions with respect to the azimuthal angle of the hardest scattering each with 

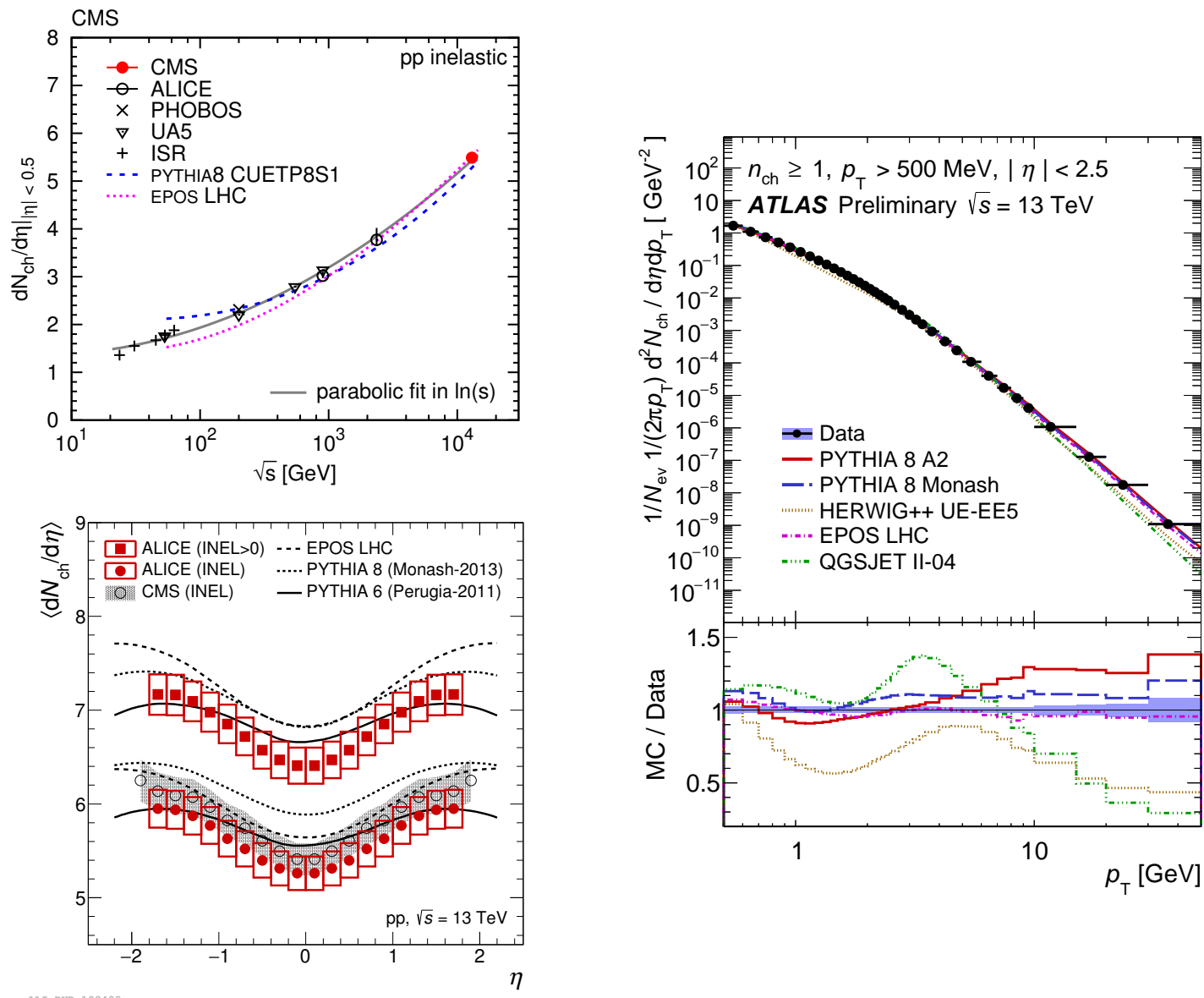

Figure 2: First measurements at $\sqrt{s}=13 \mathrm{TeV}$ by CMS [26] (top left), ALICE [27] (bottom left) and ATLAS [28] (top right).

a width of $2 / 3 \pi$, illustrated in the top left panel of Fig. 3 . To define the hardest scale, either the charged or neutral particle, jet or specific particle with the highest $p_{\mathrm{T}}$ is considered. Per region the average number of particles (number density), the $p_{\mathrm{T}}$ sum or their standard deviation is studied as a function of the $p_{\mathrm{T}}$ of the scale. Out of the various results from LHC [31-39], Fig. 3 gives examples for recent results of underlying event measurements. The bottom left panel shows the number density as a function of the transverse momentum of the leading charged track, jet or $\mathrm{Z}$ boson. It is interesting to note the smooth continuation from tracks to jets. The $\mathrm{Z}$ boson scale selects slightly different collisions at low $p_{\mathrm{T}}$ while the number density agrees at high $p_{\mathrm{T}}$ with the one from the jets. The right panel shows the measurement for the three center of mass energies measured during Run 1 of the LHC. The steep rise of the number density below $10 \mathrm{GeV} / \mathrm{c}$ is understood as a manifestation of the change of the proton-proton impact parameter in the collisions. In addition, a selection bias is present: for any non-delta function probability distribution the largest sampled value increases with the number of trials.

The underlying-event distributions are also a sensitive probe for model development and tuning and are meanwhile well described by state-of-the-art models and tunes. At the currently highest LHC energy, $\sqrt{s}=13 \mathrm{TeV}$, comparisons of detector-level (i.e. not corrected for detector efficiency) 


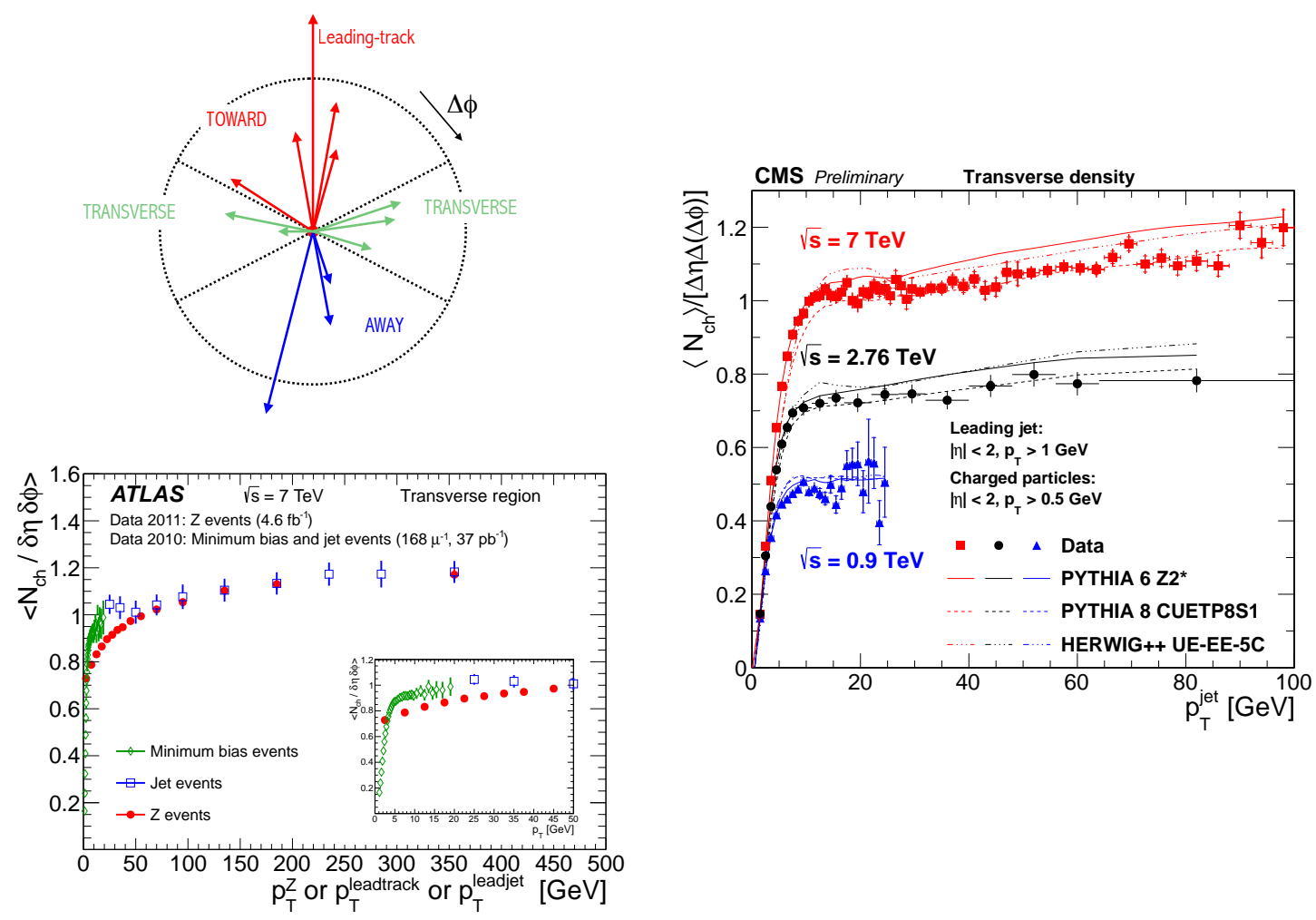

Figure 3: Definitions of underlying-event regions (top left) and measurements of the number density in the transverse region using a leading track, leading jet and leading $\mathrm{Z}$ boson (bottom left) [33] as well as for leading jets compare between three center-of-mass energies (right) [38].

underlying event distributions to current models (propagated through the detector simulation) [40] show that PYTHIA 8 with tune Monash describes the distributions well over most of the $p_{\mathrm{T}}$-range while EPOS LHC deviates by about $15 \%$.

\section{Minijets and Multiple Parton Interactions}

Jet production at high $p_{\mathrm{T}}$ is a standard QCD measurement. Measuring the particle stemming from parton-parton scatterings of a few $\mathrm{GeV} / c$ needs a different strategy, because the so-called minijet cannot be reconstructed with typical jet-reconstruction techniques due to the small number of particles. At the same time the minijet production is very abundant and proportional to the number of multiple parton interactions (MPI) occurring in the collision. In turn, the number of MPI in models has significant impact on the soft QCD probes discussed in the previous sections.

A possibility to measure the minijet contribution is to employ two-particle correlations and study the jet yields statistically. These allow to measure the so-called uncorrelated seeds [41]:

$$
\left\langle N_{\text {uncorrelated seeds }}\right\rangle=\frac{\left\langle N_{\text {trig }}\right\rangle}{\left\langle 1+N_{\text {associated,nearside }}+N_{\text {associated,awayside }}\right\rangle}
$$

where $N_{\text {trig }}$ is the number of trigger particles and $N_{\text {associated,nearside }}\left(N_{\text {associated,awayside }}\right)$ are the number of particles in the near-side (away-side) peak. The uncorrelated seeds are proportional to the num- 

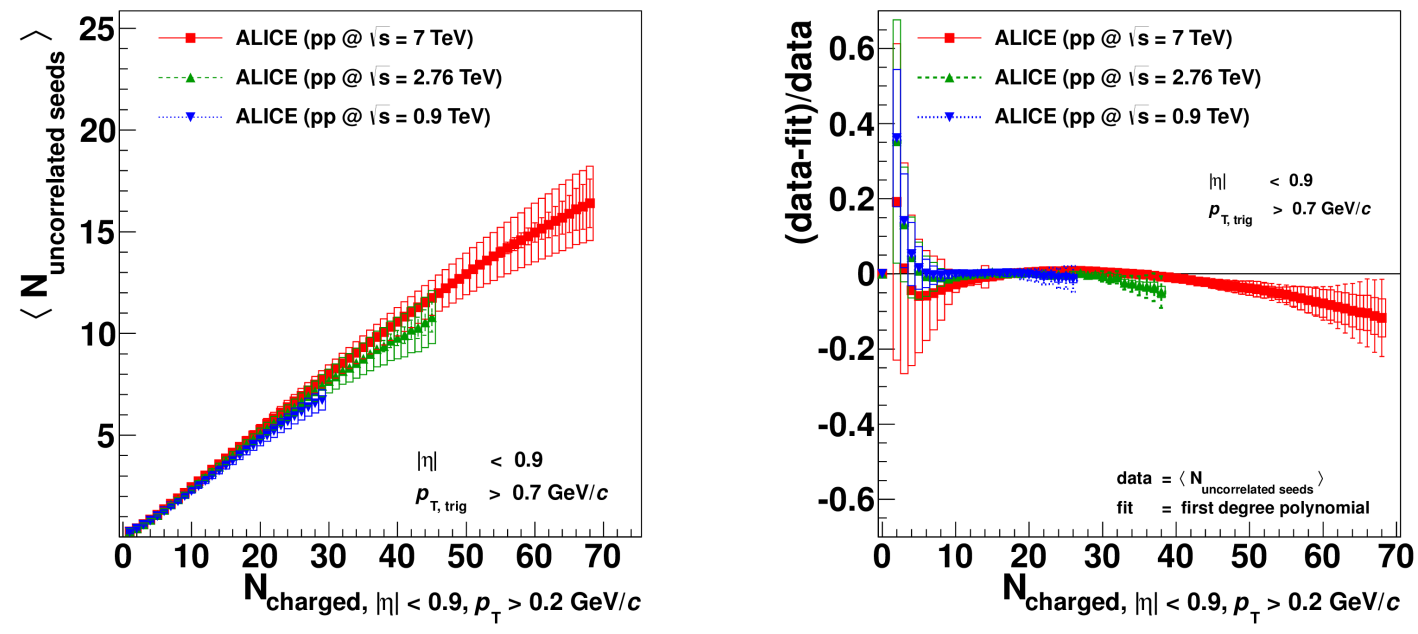

Figure 4: Number of uncorrelated seeds for three different center-of-mass energies as a function of multiplicity (left panel) and ratio of the same quantity to a linear fit illustrating the indication of a departure from linear scaling [41].

ber of MPI in MC models and can thus be used as a proxy for an MPI measurement. The left panel of Fig. 4 shows the number of uncorrelated seeds as a function of multiplicity for $\sqrt{s}=0.9,2.76$ and $7 \mathrm{TeV}$ exhibiting an almost linear growth mostly independent of center of mass energy. The right panel shows the ratio to a linear fit showing a slight departure from unity at large multiplicities. This would indicate a limit in the number of MPIs (multiplicity increases by fluctuations, not by additional MPIs) as it is found in some MC models [30]. It is interesting to continue such studies with larger event samples and at highest LHC energy.

Multiple parton interactions can also be studied with probes produced in higher-momentum parton-parton scatterings. Due to their small cross-section, such measurements are typically referred to as double parton scattering. Examples for such measurements are angular cross-section distributions of b-dijet production associated with another dijet [42] and double-charm production $[43,44]$.

\section{Identified-Particle Abundances}

The measurements discussed on the previous sections do not distinguish between particle type. The production of specific particle types is very sensitive to details of the fragmentation as well as string and color configurations. Various particle species have been measured at LHC: $\pi, \mathrm{K}^{ \pm}, \mathrm{K}^{0}$, $\mathrm{K}^{*}, \mathrm{p}, \phi, \Lambda, \Lambda^{*}, \Lambda_{b}, \Xi, \Sigma, \Omega$ as well as several $\mathrm{D}$ and $\mathrm{B}$ mesons. Despite significant tuning effort, models fail to describe the distributions for several particles, in particular (but not only) those involving strangeness. Figure 5 (left panel) presents $\Sigma$ production as a function of $p_{\mathrm{T}}$ compared to several current models where deviations up to an order of magnitude are observed. The right panel shows a measurement of the $\Lambda$ production in the underlying event which is underestimated by a factor of 2. It should be noted that EPOS LHC is reproducing the latter distribution better up to about $8 \mathrm{GeV} / c$ after which it also underestimates the data. 

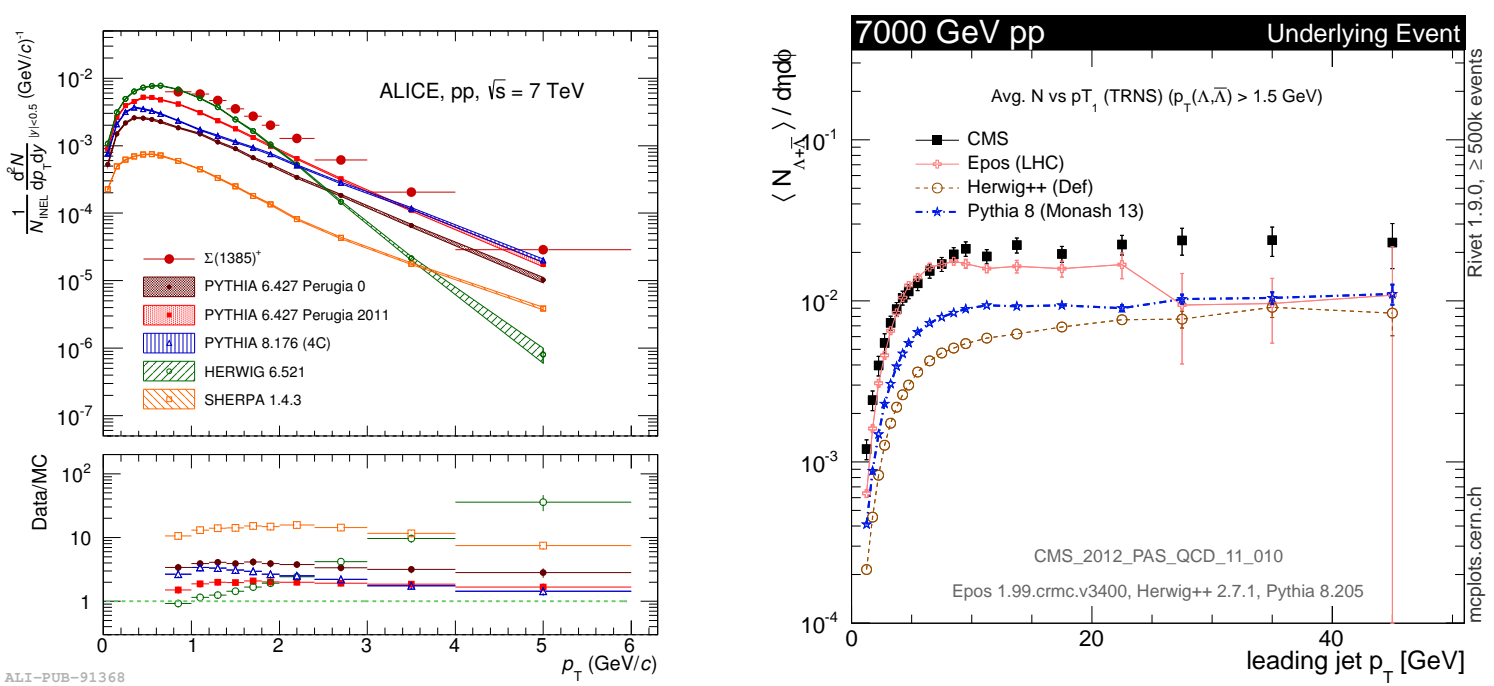

Figure 5: Strange particle yields compared to MCs. Left panel: $\Sigma(1385)^{*}$ yields as a function of $p_{\mathrm{T}}$ [45]. Right panel: $\Lambda$ production in the transverse region with respect to a leading jet $[46,47]$.

This shows that model descriptions of the production of strange baryons or strangeness in general are not accurate enough, yet. The PYTHIA model has recently been updated in this regard by improving the color-reconnection mechanism [48]. This mechanism changes the color configuration of the produced strings before hadronisation [49] which is for example needed for the correct description of the rise of the $\left\langle p_{\mathrm{T}}\right\rangle$ with multiplicity. The improved color-reconnection model includes the possibility to reconfigure two $q \bar{q}$ strings to two junctions, one connected to two $q$ and the other to two $\bar{q}$. In turn, the junctions produce baryons in the hadronisation. With this updated treatment PYTHIA succeeds to reproduce the $\Lambda / \mathrm{K}^{0}$ ratio up to about $4 \mathrm{GeV} / c$ which was underestimated by a factor of 2 before.

\section{Collectivity in Small Systems}

The surprising discovery of the near-side ridge in two-particle correlations in very high-multiplicity pp collisions in 2010 [50] (left panel of Fig. 6) stimulated significant interest. The structure is particularly interesting since it is typical in collisions of heavy ions where a Quark-Gluon Plasma (QGP) is created and is associated to collective phenomena [51]. Theoretical work aiming at a description of the phenomena therefore range from the postulation of a "mini-QGP" in pp collisions to involving gluon saturation models. The discovery of the same feature in high-multiplicity $\mathrm{p}-\mathrm{Pb}$ collisions [52] and in particular of a second ridge on the away side [53,54] (right panel of Fig. 6) made this subject a main stream topic in the study of small systems.

Nowadays, the collective nature of the observed features in $\mathrm{p}-\mathrm{Pb}$ collisions is well established based on a number of observations: the correlation structures show a particle-species dependence reminiscent of $\mathrm{Pb}-\mathrm{Pb}$ collisions [55] and involve at least 8 particles [56]. Recently, new results reported the ridge also at large rapidities [57, 58].

Recent results in pp collisions have confirmed the ridge also to be present at $13 \mathrm{TeV}[59,60]$. The magnitude is similar to $7 \mathrm{TeV}$ showing an at maximal weak center-of-mass energy dependence. 
(d) CMS N $\geq 110,1.0 \mathrm{GeV} / \mathrm{c}<\mathrm{p}_{\mathrm{T}}<3.0 \mathrm{GeV} / \mathrm{c}$

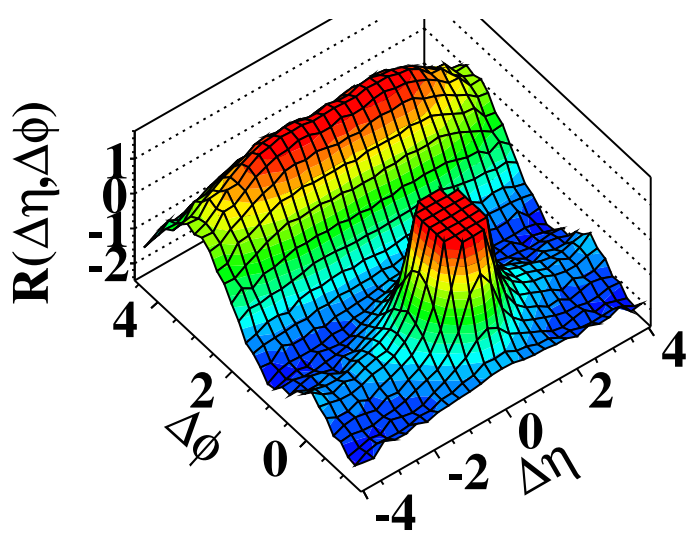

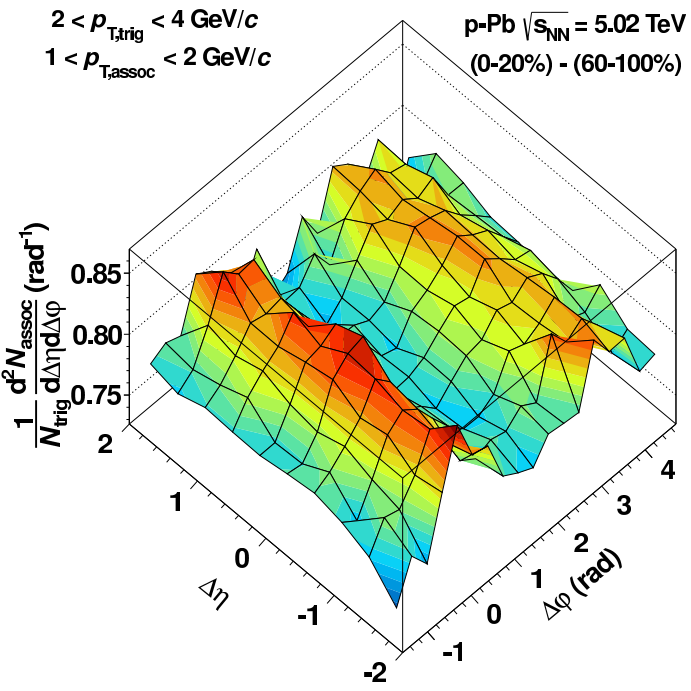

Figure 6: Ridge in pp collisions in high-multiplicity events (left panel) [50] and double ridge in high multiplicity $\mathrm{p}-\mathrm{Pb}$ collisions (right panel) [53].

To extract a signal on the away side, the dominating jet contribution has to be subtracted. Under the assumption that the jet contribution does not change in shape between low and high multiplicity collisions, it can be shown that a double ridge is also present in pp collisions [59,61].

In summary, ridge structures have been observed in $\mathrm{pp}, \mathrm{p}-\mathrm{Pb}$ and $\mathrm{Pb}-\mathrm{Pb}$ collisions hinting at similar underlying processes in the three collision systems. In that context it is interesting to note that EPOS LHC is rather successful in describing results in all three collision system and in fact includes a hydrodynamic phase in all of them. Nevertheless, it is too early to conclude that a hot and dense matter expansion is occurring also in pp collisions as this is rather new theoretical ground and there are also other processes mimicking collectivity [62]. The further study of this phenomena experimentally and theoretically has the potential for significant progress in the understanding of soft QCD.

\section{Summary}

Run I of the LHC has left a tremendous legacy of soft QCD results. Charged-particle and underlying-event distributions have challenged models at the startup of LHC but are now well described. On the contrary, identified-particle distributions and in particular strange particles still challenge models and are still not satisfactorily described. The first results from Run II at $\sqrt{s}=13 \mathrm{TeV}$ are available, where EPOS LHC and PYTHIA 8 with Monash tune made precise predictions.

Collective-like effects have been observed in high-multiplicity $\mathrm{pp}$ and $\mathrm{p}-\mathrm{Pb}$ collisions reminiscent of observations in $\mathrm{Pb}-\mathrm{Pb}$ collisions indicating that similar effects are at play in small and large collision systems. Further experimental and theoretical studies of these similarities (and potential subtle differences), as well as the search for a uniform description of the phenomena, promise significant progress in the understanding of soft QCD. 

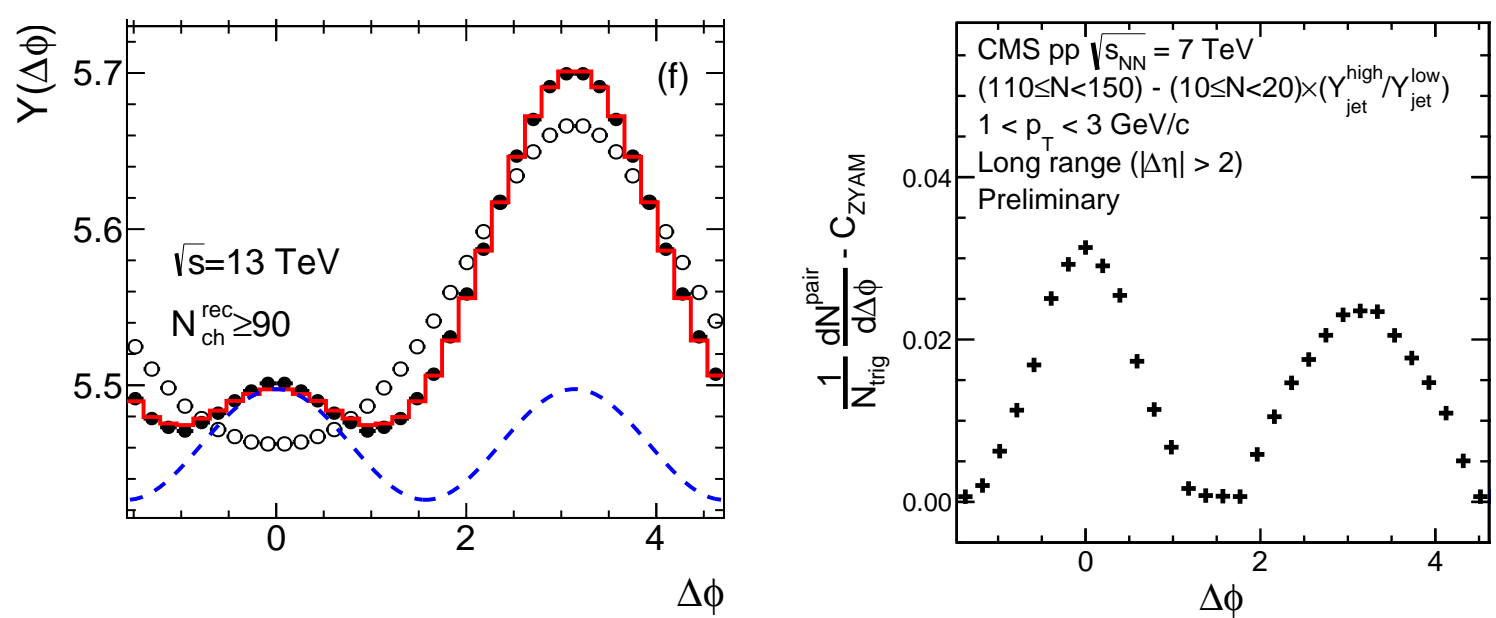

Figure 7: Double-ridge structures in pp collisions at $13 \mathrm{TeV}$ measured by ATLAS (left panel) [59] and at $7 \mathrm{TeV}$ measured by CMS (right panel) [61]

\section{References}

[1] https://twiki.cern.ch/twiki/bin/view/ALICEpublic/ALICEPublicResults

[2] https://twiki.cern.ch/twiki/bin/view/AtlasPublic

[3] http:

//cms-results.web. cern.ch/cms-results/public-results/publications

[4] https:

//Ihcb.web.cern.ch/lhcb/Physics-Results/LHCb-Physics-Results.html

[5] http://hep.fi.infn.it/LHCf/Pages/PublicationsPage.html

[6] http://totem.web.cern.ch/Totem/publ_new.html

[7] J. F. Grosse-Oetringhaus and K. Reygers, J. Phys. G 37 (2010) 083001 [arXiv:0912.0023 [hep-ex]].

[8] G. Aad et al. [ATLAS Collaboration], New J. Phys. 13 (2011) 053033 [arXiv:1012.5104 [hep-ex]].

[9] G. Aad et al. [ATLAS Collaboration], Phys. Lett. B 688 (2010) 21 [arXiv:1003.3124 [hep-ex]].

[10] V. Khachatryan et al. [CMS Collaboration], JHEP 1002 (2010) 041 [arXiv:1002.0621 [hep-ex]].

[11] V. Khachatryan et al. [CMS Collaboration], Phys. Rev. Lett. 105 (2010) 022002 [arXiv:1005.3299 [hep-ex]].

[12] V. Khachatryan et al. [CMS Collaboration], JHEP 1101 (2011) 079 [arXiv:1011.5531 [hep-ex]].

[13] S. Chatrchyan et al. [CMS Collaboration], JHEP 1108 (2011) 086 [arXiv:1104.3547 [hep-ex]].

[14] R. Aaij et al. [LHCb Collaboration], Eur. Phys. J. C 72 (2012) 1947 [arXiv:1112.4592 [hep-ex]].

[15] R. Aaij et al. [LHCb Collaboration], Eur. Phys. J. C 74 (2014) 5, 2888 [arXiv:1402.4430 [hep-ex]].

[16] K. Aamodt et al. [ALICE Collaboration], Eur. Phys. J. C 65 (2010) 111 [arXiv:0911.5430 [hep-ex]].

[17] K. Aamodt et al. [ALICE Collaboration], Eur. Phys. J. C 68 (2010) 89 [arXiv:1004.3034 [hep-ex]].

[18] K. Aamodt et al. [ALICE Collaboration], Eur. Phys. J. C 68 (2010) 345 [arXiv:1004.3514 [hep-ex]]. 
[19] K. Aamodt et al. [ALICE Collaboration], Phys. Lett. B 693 (2010) 53 [arXiv:1007.0719 [hep-ex]].

[20] B. B. Abelev et al. [ALICE Collaboration], Eur. Phys. J. C 73 (2013) 12, 2662 [arXiv:1307.1093 [nucl-ex]].

[21] B. B. Abelev et al. [ALICE Collaboration], Phys. Lett. B 727 (2013) 371 [arXiv:1307.1094 [nucl-ex]].

[22] G. Antchev et al. [TOTEM Collaboration], Europhys. Lett. 98 (2012) 31002 [arXiv:1205.4105 [hep-ex]].

[23] S. Chatrchyan et al. [CMS and TOTEM Collaborations], Eur. Phys. J. C 74 (2014) 10, 3053 [arXiv:1405.0722 [hep-ex]].

[24] G. Antchev et al. [TOTEM Collaboration], Eur. Phys. J. C 75 (2015) 3, 126 [arXiv:1411.4963 [hep-ex]].

[25] J. M. Katzy, Prog. Part. Nucl. Phys. 73 (2013) 141.

[26] V. Khachatryan et al. [CMS Collaboration], Phys. Lett. B 751 (2015) 143 [arXiv:1507.05915 [hep-ex]].

[27] J. Adam et al. [ALICE Collaboration], arXiv:1509.08734 [nucl-ex].

[28] ATLAS Collaboration, Charged-particle distributions in $\operatorname{sqrt}(\mathrm{s})=13 \mathrm{TeV}$ pp interactions measured with the ATLAS detector at the LHC, ATLAS-CONF-2015-028

[29] T. Pierog, I. Karpenko, J. M. Katzy, E. Yatsenko and K. Werner, Phys. Rev. C 92 (2015) 3, 034906 [arXiv:1306.0121 [hep-ph]].

[30] P. Skands, S. Carrazza and J. Rojo, Eur. Phys. J. C 74 (2014) 8, 3024 [arXiv:1404.5630 [hep-ph]].

[31] G. Aad et al. [ATLAS Collaboration], Phys. Rev. D 83 (2011) 112001 [arXiv:1012.0791 [hep-ex]].

[32] G. Aad et al. [ATLAS Collaboration], Eur. Phys. J. C 71 (2011) 1636 [arXiv:1103.1816 [hep-ex]].

[33] G. Aad et al. [ATLAS Collaboration], Eur. Phys. J. C 74 (2014) 12, 3195 [arXiv:1409.3433 [hep-ex]].

[34] S. Chatrchyan et al. [CMS Collaboration], Eur. Phys. J. C 72 (2012) 2080 [arXiv:1204.1411 [hep-ex]].

[35] V. Khachatryan et al. [CMS Collaboration], Eur. Phys. J. C 70 (2010) 555 [arXiv:1006.2083 [hep-ex]].

[36] S. Chatrchyan et al. [CMS Collaboration], JHEP 1304 (2013) 072 [arXiv:1302.2394 [hep-ex]].

[37] S. Chatrchyan et al. [CMS Collaboration], JHEP 1109 (2011) 109 [arXiv:1107.0330 [hep-ex]].

[38] V. Khachatryan et al. [CMS Collaboration], JHEP 1509 (2015) 137 [arXiv:1507.07229 [hep-ex]].

[39] B. Abelev et al. [ALICE Collaboration], JHEP 1207 (2012) 116 [arXiv:1112.2082 [hep-ex]].

[40] ATLAS Collaboration, Detector level leading track underlying event distributions at $13 \mathrm{TeV}$ measured in ATLAS, ATL-PHYS-PUB-2015-019

[41] B. Abelev et al. [ALICE Collaboration], JHEP 1309 (2013) 049 [arXiv:1307.1249 [nucl-ex]].

[42] CMS Collaboration, Studies of 2 b-jet +2 jet production in proton-proton collisions at $7 \mathrm{TeV}, \mathrm{CMS}$ PAS FSQ-13-010

[43] R. Aaij et al. [LHCb Collaboration], JHEP 1206 (2012) 141 [JHEP 1403 (2014) 108] [arXiv:1205.0975 [hep-ex]].

[44] R. Aaij et al. [LHCb Collaboration], Phys. Lett. B 707 (2012) 52 [arXiv:1109.0963 [hep-ex]]. 
[45] B. B. Abelev et al. [ALICE Collaboration], Eur. Phys. J. C 75 (2015) 1, 1 [arXiv:1406.3206 [nucl-ex]].

[46] S. Chatrchyan et al. [CMS Collaboration], Phys. Rev. D 88 (2013) 052001 [arXiv:1305.6016 [hep-ex]].

[47] http://mcplots.cern.ch

[48] J. R. Christiansen, arXiv:1510.04859 [hep-ph].

[49] G. Gustafson, Acta Phys. Polon. B 40 (2009) 1981 [arXiv:0905.2492 [hep-ph]].

[50] V. Khachatryan et al. [CMS Collaboration], JHEP 1009 (2010) 091 [arXiv:1009.4122 [hep-ex]].

[51] U. Heinz and R. Snellings, Ann. Rev. Nucl. Part. Sci. 63 (2013) 123 [arXiv:1301.2826 [nucl-th]].

[52] S. Chatrchyan et al. [CMS Collaboration], Phys. Lett. B 718 (2013) 795 [arXiv:1210.5482 [nucl-ex]].

[53] B. Abelev et al. [ALICE Collaboration], Phys. Lett. B 719 (2013) 29 [arXiv:1212.2001 [nucl-ex]].

[54] G. Aad et al. [ATLAS Collaboration], Phys. Rev. Lett. 110 (2013) 18, 182302 [arXiv:1212.5198 [hep-ex]].

[55] B. B. Abelev et al. [ALICE Collaboration], Phys. Lett. B 726 (2013) 164 [arXiv:1307.3237 [nucl-ex]].

[56] V. Khachatryan et al. [CMS Collaboration], Phys. Rev. Lett. 115 (2015) 1, 012301 [arXiv:1502.05382 [nucl-ex]].

[57] J. Adam et al. [ALICE Collaboration], arXiv:1506.08032 [nucl-ex].

[58] LHCb Collaboration, First measurements of long-range near-side angular correlations in $\sqrt{s_{\mathrm{NN}}}=5 \mathrm{TeV}$ proton-lead collisions in the forward region, LHCB-CONF-2015-004

[59] G. Aad et al. [ATLAS Collaboration], arXiv:1509.04776 [hep-ex].

[60] V. Khachatryan et al. [CMS Collaboration], arXiv:1510.03068 [nucl-ex].

[61] CMS Collaboration, Azimuthal anisotropy harmonics from long-range correlations in high multiplicity pp collisions at sqrt(s) $=7 \mathrm{TeV}$, CMS-PAS-HIN-15-009

[62] A. Ortiz Velasquez, P. Christiansen, E. Cuautle Flores, I. Maldonado Cervantes and G. Paï̈̆̆, Phys. Rev. Lett. 111 (2013) 4, 042001 [arXiv:1303.6326 [hep-ph]]. 